\section{A study on the comparative values placed on consultations by pet owners and primary care veterinary surgeons in the UK}

\section{Thomas Theobald, Mark Dunning}

School of Veterinary Medicine and Science, University of Nottingham, Leicestershire, UK

To date there is limited literature investigating the veterinary surgeon-pet owner relationship in the UK. It is suspected to mirror human medicine, where relationship centred care leads to high levels of satisfaction with an emphasis on empathy. In contrast to human medicine, pet owners are free to choose their veterinary surgery and therefore factors influencing this relationship are particularly important to optimise their experience.

The aim was to identify what UK pet owners the value during a consultation with their veterinary surgeon and what determines whether they return to the see the same veterinary surgeon on subsequent occasions. A further aim was to compare the client values with those of the veterinary surgeons.

The study was completed using two questionnaires one for pet owners and one for veterinary surgeons. The respective questionnaires were designed using the Calgary-Cambridge consultation model and amended where necessary to optimise their utility for owners or veterinary surgeons. A pilot study was sent to a small number of pet owners and veterinary surgeons and their comments used to refine the questions. The final questionnaires were sent to veterinary practices throughout the UK to be completed by owners and veterinary surgeons.

A total of 112 owners and 360 veterinary surgeons completed questionnaires. The results were analysed and grouped into categories according to the 'generic' response that had been given. Responses were qualitatively analysed and the top 10 categories determined according to free text phrasing. The most important aspect of the consultation for the owner was the veterinary surgeon 'engaging with their animal' (22\%), the 'client-vet relationship' appeared the least important (6\%). Most pet owners felt that their expectations prior to the consultation were met at the end (95\%). The veterinary surgeons felt communication was the most important $(41 \%)$, the least important being the clinical examination (11\%). The most common reason pet owners returned to the same Veterinary practice was for continuity (25\%). In contrast, veterinary surgeons felt trust was the most important factor influencing owners returning (53\%). Both owners and veterinary surgeons placed high value on 'explanation and planning' during the consultation. Overall there are differences in what pet owners and veterinary surgeons feel are most important during the consultation. This information could aid with improving overall pet owner satisfaction in primary care practice.

\section{Presentation and management of dystocia in dogs attending emergency-care veterinary practices in the UK}

\section{Erin Manson', Dave Brodbelt', Amanda Boag', David Church', Paul McGreevy ${ }^{3}$, Aoife O'Sullivan², Peter Thomson ${ }^{3}$, Dan O'Neill'1}

The Royal Veterinary College, Hatfield, Hertfordshire, UK VetsNow Ltd, Dunfermline, Fife, UK

The University of Sydney, Sydney, NSW, Australia

Canine dystocia generally requires urgent intervention and can often occur outside of routine opening hours for primary-care veterinary practices. Greater understanding of the frequency, background and clinical management of dystocic bitches treated in emergency-care practices could inform veterinary advice for breeders and owners. The current study aimed to report on prevalence, case management and outcomes of dystocia in bitches attending emergency-care practices in the UK.

This study used electronic patient record data shared from September 1st 2012 to February 14th, 2014 from 50 VetsNow clinics participating in the VetCompass project. The free-text clinical notes and the VeNom diagnosis fields were searched for possible dystocia cases that were verified manually. Additional information extracted on cases included date of dystocia, parity, prior dystocia, diagnostic methods, clinical management and bitch survival.

There were 685 cases of dystocia, giving a prevalence of $2.3 \%$ (95\% confidence interval (CI): $2.1-2.4 \%$ ) of the emergency care caseload. Among the purebred bitches $(n=615$; $94.2 \%)$, the most common breeds presented were Chihuahua $(11 \%)$, Staffordshire Bull Terrier (8.3\%), Jack Russell Terrier (6.3\%), Pug (6.1\%) and French Bulldog (4.1\%). The median bodyweight of dystocic bitches was $10.5 \mathrm{~kg}$ (interquartile range (IQR): $6.3-23.4 \mathrm{~kg}$ ), and the median age at presentation was 3.0 years (IQR: $2.0-4.0$ years). Overall, $185(27.1 \%)$ of cases underwent diagnostic imaging and 119 (54.6\%) were multiparous, of which $34(28.6 \%)$ reported a previous dystocia. Of the 377 (55\%) cases that received oxytocin (not including administration after caesarean section (CS)), 136 (36.1\%) received 2 or more injections and 121 (32.1\%) later underwent CS. There were $337(49.2 \%)$ cases that underwent CS, with 104 (38.1\%) of these surgeries including an ovariohysterectomy. The median count of puppies (live and dead) per litter was 4 (IQR: 3-6, range: $0-17)$ and median live litter size at discharge was 3 (IQR: 2-5, range: 0-14). Overall, 11 (1.6\%) of bitches died (unassisted or euthanasia) during or immediately after their dystocia. The most commonly reported causes of dystocia were foetal malposition (89 cases, 34.5\%), foeto-maternal disproportion (77 cases, $29.8 \%$ ) and uterine inertia (75 cases, 29.1\%)

This study highlights canine dystocia as an important emergency presentation that often resulted in surgery. These results will help clinicians to benchmark their clinical decisionmaking and to provide reliable prognostic advice for owners. 